Pacific Journal of Mathematic 


\section{CHAINABLE CONTINUA AND INDECOMPOSABILITY}

\section{E. Burgess}

This paper includes a study of continua ${ }^{1}$ which are both linearly chainable and circularly chainable. Since there exist indecomposable continua and 2 indecomposable continua which are linearly chainable, it follows from Theorem 7 that there exist indecomposable continua and decomposable continua which have both of these types of chainability.

A linear chain $C$ is a finite collection of open sets $L_{1}, L_{2}, \cdots, L_{n}$ such that

(1) each element of $C$ contains an open set that does not intersect any other element of $C$,

(2) $\rho\left(L_{i}, L_{j}\right)>0$ if $|i-j|>1$, and

(3) $L_{i} \cdot L_{j} \neq 0$ if $|i-j| \leq 1$. If this is modified so that $L_{1} \cdot L_{n} \neq 0$, then $C$ is called a circular chain. Each of the sets $L_{1}, L_{2}, \cdots, L_{n}$ is called a link of $C$, and $C$ is sometimes denoted by $\left(L_{1}, L_{2}, \cdots, L_{n}\right)$ or $C\left(L_{1}, L_{2}, \cdots, L_{n}\right)$. If $\varepsilon$ is a positive number and $C$ is a linear chain such that each link of $C$ has a diameter less than $\varepsilon$, then $C$ is called a linear $\varepsilon$-chain. A circular $\varepsilon$-chain is defined similarly.

If $C$ is either a linear chain or a circular chain and $H_{1}, H_{2}, \cdots, H_{n}$ are connected sets covered by $C$, then these sets are said to have the order $H_{1}, H_{2}, \cdots, H_{n}$ in $C$ provided (1) no link of $C$ intersects two of these $n$ sets and (2) for each $i(i<n)$, there is a linear sub-chain in $C$ which covers $H_{i}+H_{i+1}$ and which does not intersect any other of the sets $H_{1}, H_{2}, \cdots, H_{n}$.

A continuum $M$ is said to be linearly chainable $e^{2}$ if for every positive number $\varepsilon$, there is a linear $\varepsilon$-chain covering $M$. A continuum $M$ is said to be circularly chainable if for every positive number $\varepsilon$, there is a circular $\varepsilon$-chain covering $M$.

A tree $T$ is a finite coherent ${ }^{3}$ collection of open sets such that

(1) each element of $T$ contains an open set that does not intersect any other element of $T$,

(2) each two nonintersecting elements of $T$ are a positive distance apart, and

(3) no subcollection of $T$ consisting of more than two elements is a circular chain. If $\varepsilon$ is a positive number and $T$ is a tree such that

Presented to the American Mathematical Society, August 29, 1957; received by the editors December 5, 1958. This work was supported by the National Science Foundation under G-2574 and G-5880. Most of these results were obtained while the author was a visiting lecturer at the University of Wisconsin.

1 Throughout this paper, a connected compact metric space is called a continuum.

2 In some places in the literature, such continua have been said to be chainable.

${ }^{3}$ A collection $G$ of sets is said to be coherent if for any two subcollections $G_{1}$ and $G_{2}$ of $G$ such that $G_{1}+G_{2}=G$, some element of $G_{1}$ intersects some element of $G_{2}$. 
each element of $T$ has a diameter less than $\varepsilon$, then $T$ is called an $\varepsilon$-tree. A continuum $M$ is said to be tree-like if for every positive number $\varepsilon$, there is an $\varepsilon$-tree covering $M$.

A continuum $M$ is said to be the essential sum of the elements of a collection $G$ if the sum of the elements of $G$ is $M$ and no element of $G$ is a subset of the sum of the other elements of $G$. If $n$ is a positive integer and the continuum $M$ is the essential sum of $n$ continua and is not the essential sum of $n+1$ continua, then $M$ is said to be $n$-indecomposable. ${ }^{4}$

A continuum $M$ is said to be unicoherent if the intersection of each two continua having $M$ as their sum is a continuum. A continuum $M$ is said to be bicoherent if for any two proper subcontinua $M_{1}$ and $M_{2}$ having $M$ as their sum, the set $M_{1} \cdot M_{2}$ is the sum of two continua that do not intersect.

A continuum $M$ is said to be a triod if $M$ is the essential sum of three continua such that their intersection is a continuum which is the intersection of each two of them.

THEOREM 1. If the continuum $M$ is either linearly chainable or circularly chainable, then $M$ does not contain a triod. ${ }^{5}$

Proof. Since it is easy to see that every proper subcontinuum of $M$ is linearly chainable, it will be sufficient to show that $M$ is not a triod.

Suppose that $M$ is a triod. Let $M_{1}, M_{2}$, and $M_{3}$ be three continua having $M$ as their essential sum such that their intersection is a continuum $H$ which is the intersection of each two of them. For each $i(i \leq 3)$, let $p_{i}$ be a point of $M_{i}$ that is not in either of the other two of the continua $M_{1}, M_{2}$, and $M_{3}$. Let $\varepsilon$ be a positive number which is less than each of the numbers $\rho\left(p_{1}, M_{2}+M_{3}\right), \rho\left(p_{2}, M_{1}+M_{3}\right)$, and $\rho\left(p_{3}, M_{1}+M_{2}\right)$. Let $C$ be either a linear $\varepsilon$-chain or a circular $\varepsilon$-chain which covers $M$. Since no link of $C$ intersects two of the sets $p_{1}, p_{2}, p_{3}$, and $H$, consider the case in which these four sets are in $C$ in the order named. This would involve the contradiction that $M_{2}$ intersects either the link of $C$ that contains $p_{1}$ or the link of $C$ that contains $p_{3}$. A similar contradiction results from supposing any other order of the sets $p_{1}, p_{2}, p_{3}$, and $H$ in $C$.

THEOREM 2. If the unicoherent continuum $M$ is not a triod and $M_{1}, M_{2}, M_{3}$ are three continua having $M$ as their essential sum, then

${ }^{4}$ For any such continuum $M$, there is a unique collection consisting of $n$ indecomposable continua having $M$ as their essential sum [4].

${ }_{5}^{5}$ Bing [2] has used the fact that no linearly chainable continuum contains a triod, but for completeness a proof is given here for both types of chainability. 
some two of these continua do not intersect and the other one intersects each of these two in a continuum.

Proof. Suppose that each two of the continua $M_{1}, M_{2}$, and $M_{3}$ intersect. It follows from the unicoherence of $M$ that each of the sets $M_{1} \cdot\left(M_{2}+M_{3}\right)$ and $M_{2} \cdot\left(M_{1}+M_{3}\right)$ is a continuum and their sum is a continuum. Let $N=M_{1} \cdot\left(M_{2}+M_{3}\right)+M_{2} \cdot\left(M_{1}+M_{3}\right)=M_{1} \cdot M_{2}+M_{1} \cdot M_{3}$ $+M_{2} \cdot M_{3}$. Hence $M$ is the essential sum of the three continua $M_{1}+N$, $M_{2}+N$, and $M_{3}+N$ such that $N$ is the intersection of each two of them and the intersection of all three of them. Since this is contrary to the hypothesis that $M$ is not a triod, it follows that some two of the continua $M_{1}, M_{2}$, and $M_{3}$ do not intersect. Consider the case in which $M_{1}$ and $M_{3}$ do not intersect. Then $M_{2}$ intersects both $M_{1}$ and $M_{3}$, and since $M_{1} \cdot M_{2}=M_{1} \cdot\left(M_{2}+M_{3}\right)$ and $M_{3} \cdot M_{2}=M_{3} \cdot\left(M_{2}+M_{1}\right)$, it follows from the unicoherence of $M$ that each of the sets $M_{1} \cdot M_{2}$ and $M_{3} \cdot M_{2}$ is a continuum.

THeorem 3. If the unicoherent continuum $M$ is circularly chainable, then $M$ is either indecomposable or 2-indecomposable.

Proof. Suppose that $M$ is the essential sum of three continua $M_{1}$, $M_{2}$, and $M_{3}$. By Theorem $1, M$ is not a triod. Hence by Theorem 2, one of these three continua, say $M_{2}$, intersects each of the other two such that $M_{1} \cdot M_{2}$ and $M_{2} \cdot M_{3}$ are continua and $M_{1}$ does not intersect $M_{3}$. For each $i(i \leq 3)$, let $p_{i}$ be a point of $M_{i}$ which is not in either of the other two of the continua $M_{1}, M_{2}$, and $M_{3}$. Let $\varepsilon$ be a positive number which is less than each of the numbers $\rho\left(p_{1}, M_{2}+M_{3}\right), \rho\left(p_{2}, M_{1}+M_{3}\right)$, $\rho\left(p_{3}, M_{1}+M_{2}\right)$, and $\rho\left(M_{1}, M_{3}\right)$. Let $C$ be a circular $\varepsilon$-chain which covers $M$. A contradiction can be obtained as follows for each of the three types of order in $C$ for the five sets $p_{1}, p_{2}, p_{3}, M_{2} \cdot M_{1}$, and $M_{2} \cdot M_{3}$.

Case 1. If these five sets have the order $p_{i}, p_{j}, p_{k}, M_{2} \cdot M_{1}, M_{2} \cdot M_{3}$ in $C$, then $M_{j}$ would intersect a link of $C$ that contains one of the points $p_{i}$ and $p_{k}$, contrary to the choice of $\varepsilon$.

Case 2. If these five sets have the order $p_{1}, M_{2} \cdot M_{1}, p_{i}, p_{i}, M_{2} \cdot M_{3}$ in $C$, then $M_{2}$ would intersect a link of $C$ that contains one of the points $p_{1}$ and $p_{3}$, contrary to the choice of $\varepsilon$.

Case 3. If these five sets have the order $p_{2}, M_{2} \cdot M_{1}, p_{i}, p_{j}, M_{2} \cdot M_{3}$ in $C$, then each link of one of the linear chains of $C$ from $p_{1}$ to $p_{3}$ would lie in $M_{1}+M_{3}$. This would involve the contradiction that some link of $C$ intersects both $M_{1}$ and $M_{3}$.

THEOREM 4. If the circularly chainable continuum $M$ is separated 
by one of its subcontinua, then $M$ is linearly chainable.

Proof. Let $K$ be a subcontinuum of $M$ which separates $M$. Then $M$ is the sum of two continua $M_{1}$ and $M_{2}$ such that $K$ is their intersection. Let $p_{1}$ and $p_{2}$ be points of $M_{1}-K$ and $M_{2}-K$, respectively, let $\varepsilon$ be a positive number less than each of the numbers $\rho\left(p_{1}, M_{2}\right)$ and $\rho\left(p_{2}, M_{1}\right)$, and let $C$ be a circular $\varepsilon$-chain covering $M$. Then each link of one of the linear chains in $C$ from $p_{1}$ to $p_{2}$ is a subset of $M-K$. Let $L_{1}, L_{2}, \cdots, L_{n}$ be the links of $C$ such that $L_{1}$ contains $p_{1}$ and there is a positive integer $r$ such that $L_{r}$ contains $p_{2}$ and no link of the linear chain $\left(L_{1}, L_{2}, \cdots, L_{r}\right)$ intersects $K$. There exist integers $i$ and $j$ such that $L_{i}$ is the first link of $\left(L_{1}, L_{2}, \cdots, L_{r}\right)$ which intersects $M_{2}$ and $L_{j}$ is the last link of $\left(L_{1}, L_{2}, \cdots, L_{r}\right)$ which intersects $M_{1}$. Then $\left(M_{2} \cdot L_{i}, M_{2} \cdot L_{i+1}\right.$, $\left.\cdots, M_{2} \cdot L_{r}, L_{r+1}, \cdots, L_{n}, M_{1} \cdot L_{1}, M_{1} \cdot L_{2}, \cdots, M_{1} \cdot L_{j}\right)$ is a linear $\varepsilon$-chain covering $M$.

THEOREM 5. Every circularly chainable continuum $M$ is either unicoherent or bicoherent. Furthermore, $M$ is unicoherent provided some subcontinuum of $M$ separates $M$, and $M$ is bicoherent provided no subcontinuum of $M$ separates $M$.

Proof. Suppose that $M$ is the sum of two continua $H$ and $K$ such that $H \cdot K$ is the sum of three mutually separated sets $Y_{1}, Y_{2}$, and $Y_{3}$. There exist three open sets $D_{1}, D_{2}$, and $D_{3}$ containing $Y_{1}, Y_{2}$, and $Y_{3}$, respectively, such that the closures of $D_{1}, D_{2}$, and $D_{3}$ are disjoint. For each $i(i \leq 3)$, there exists a subcontinuum $K_{i}$ of $K$ irreducible from $Y_{i}$ to $M-D_{i}$. The continuum $H+K_{1}+K_{2}+K_{3}$ is a triod, and this is contrary to Theorem 1. Hence it follows that if $M_{1}$ and $M_{2}$ are two continua having $M$ as their sum, then the set $M_{1} \cdot M_{2}$ is either a continuum or the sum of two continua.

It follows from Theorem 4 that $M$ is linearly chainable, and hence unicoherent [3], provided some subcontinuum of $M$ separates $M$. From this and the argument in the previous paragraph, it follows that $M$ is bicoherent provided no subcontinuum of $M$ separates $M$.

THEOREM 6. If the circularly chainable continuum $M$ is irreducible about some finite set consisting of $n$ points, then there is a positive integer $k$ not greater than $n$ such that $M$ is $k$-indecomposable.

Proof. By Theorem 5, $M$ is either unicoherent or bicoherent. If $M$ is unicoherent, it follows from Theorem 3 that $M$ is either indecomposable or 2-indecomposable. If $M$ is bicoherent, it follows from Corollary 6.1 of [5] that there is a positive integer $k$ not greater than $n$ such that $M$ is $k$-indecomposable. 
Theorem 7. If the continuum $M$ is linearly chainable, then in order that $M$ should be circularly chainable, it is necessary and sufficient that $M$ be either indecomposable of 2-indecomposable.

Proof of necessity. Since every lineary chainable continuum is unicoherent [3], it follows from Theorem 3 that $M$ is either indecomposable or 2-indecomposable.

Proof of sufficiency. The case where $M$ is indecomposable and the case where $M$ is 2 -indecomposable will be considered separately.

Case 1. Suppose $M$ is indecomposable, and let $C\left(L_{1}, L_{2}, \cdots, L_{n}\right)$ be a linear $\varepsilon$-chain covoring $M$. There exist two disjoint continua $K_{1}$ and $K_{2}$ of $M$ such that each of them intersects each of the sets $L_{1}-\operatorname{cl}\left(L_{2}\right)$ and $L_{n}-\operatorname{cl}\left(L_{n-1}\right)$. If follows that there exist a positive number $\varepsilon^{\prime}$, a linear $\varepsilon^{\prime}$-chain $C^{\prime}$ covering $M$, and two subchains $C_{1}$ and $C_{2}$ of $C^{\prime}$ such that

(1) each link of $C^{\prime}$ is a subset of some link of $C$,

(2) $C_{1}$ and $C_{2}$ have no common link, and

(3) each of the chains $C_{1}$ and $C_{2}$ has one end link in $L_{1}-\operatorname{cl}\left(L_{2}\right)$ and the other end link in $L_{n}-\operatorname{cl}\left(L_{n-1}\right)$. Let $W_{1}$ denote the set of all points of $M$ that are covered by $C_{1}$ and let $W_{2}$ denote $M-W_{1}$. Then $\left(L_{1}, W_{1} \cdot L_{2}, W_{1} \cdot L_{3}, \cdots, W_{1} \cdot L_{n-1}, L_{n}, W_{2} \cdot L_{n-1}, W_{2} \cdot L_{n-2}, \cdots, W_{2} \cdot L_{2}\right)$ is a circular $\varepsilon$-chain covering $M$.

Case 2. If $M$ is 2-indecomposable, there exist two indecomposable continua $M_{1}$ and $M_{2}$ such that $M$ is their essential sum and $M_{1} \cdot M_{2}$ is a continuum. Let $\varepsilon$ be a positive number. There exists a linear $\varepsilon$-chain $C$ covering $M$ such that $M_{1}$ intersects $L_{1}-c l\left(L_{2}\right)$ and $M_{2}$ intersects $L_{n}-\operatorname{cl}\left(L_{n-1}\right)$. Since each composant of $M_{i}(i=1,2)$ is everywhere dense in $M_{i}$, it follows that for each $i(i=1,2)$ there exist two disjoint subcontinua $K_{i}$ and $H_{i}$ of $M_{i}$ such that

(1) each of them intersects each link of $C$ that intersects $M_{i}$,

(2) $H_{i}$ contains $M_{1} \cdot M_{2}$,

(3) each of the continua $H_{1}$ and $K_{1}$ intersects $L_{1}-c l\left(L_{2}\right)$, and

(4) each of the continua $H_{2}$ and $H_{2}$ intersects $L_{n}-c l\left(L_{n-1}\right)$. Hence there exist a positive number $\varepsilon^{\prime}$, a linear $\varepsilon^{\prime}$-chain $C^{\prime}$ covering $M$, and three subchains $C_{1}, C_{2}$, and $C_{3}$ of $C^{\prime}$ such that

(1) each link of $C^{\prime}$ is a subset of a link of $C$,

(2) no two of the chains $C_{1}, C_{2}$, and $C_{3}$ have a common link,

(3) one end link of $C_{1}$ is in $L_{1}-\operatorname{cl}\left(L_{2}\right)$,

(4) one end link of $C_{2}$ is in $L_{n}-\operatorname{cl}\left(L_{n-1}\right)$,

(5) some link of $C$ contains a link of $C_{1}$ and a link of $C_{2}$, and 
(6) $C_{3}$ has one end link in $L_{1}-\operatorname{cl}\left(L_{2}\right)$ and the other end link in $L_{n}-\operatorname{cl}\left(L_{n-1}\right)$. Let $W$ denote the set of all points of $M$ that are covered by $C_{3}$, and let $Y$ denote $M-W$. Then $\left(L_{1}, W \cdot L_{2}, W \cdot L_{3}, \cdots, W \cdot L_{n-1}\right.$, $\left.L_{n}, Y \cdot L_{n-1}, Y \cdot L_{n-2}, \cdots, Y \cdot L_{2}\right)$ is a circular $\varepsilon$-chain covering $M$.

THEOREM 8. If $n$ is a positive integer and for each proper subcontinuum $H$ of the continuum $M$ there is a positive integer $r$ not greater than $n$ such that $H$ is $r$-indecomposable, then there is a positive integer $k$ not greater than $n$ such that $M$ is $k$-indecomposable.

Proof. Suppose that $M$ is the essential sum of $n+1$ continua $M_{1}, M_{2}, \cdots, M_{n+1}$. Some $n$ of these continua have a connected sum, so consider the case in which $M_{2}+M_{3} \cdots+M_{n+1}$ is connected. There is an open set $D$ which intersects $M_{1}$ such that the closure of $D$ does not intersect $M_{2}+M_{3}+\cdots+M_{n+1}$. There is a subcontinuum $M_{1}^{\prime}$ of $M_{1}$ irreducible from the closure of $D$ to $M_{2}+M_{3}+\cdots+M_{n+1}$. This involves the contradiction that $M_{1}^{\prime}+M_{2}+M_{3}+\cdots+M_{n+1}$ is a proper subcontinuum of $M$ and is the essential sum of $n+1$ continua.

THEOREM 9. If every proper subcontinuum of the continuum $M$ is circularly chainable, then every subcontinuum of $M$ is either indecomposable or 2-indecomposable.

Proof. Since each proper subcontinuum of $M$ is a proper subcontinuum of another proper subcontinuum of $M$, it follows that every proper subcontinuum of $M$ is linearly chainable. Hence by Theorem 7, every proper subcontinuum of $M$ is either indecomposable or 2-indecomposable. Consequently, it follows from Theorem 8 that $M$ itself is either indecomposable or 2-indecomposable.

EXAMPLES. A pseudo-arc $[1 ; 6]$ is an example of an indecomposable continuum which satisfies the hypothesis of Theorem 9 , and a continuum which is the sum of two pseudo-ares with a point as their intersection is an example of a 2-indecomposable continuum which satisfies this hypothesis.

THEOREM 10. If the tree-like continuum $M$ is circularly chainable, then $M$ is linearly chainable.

Pooof. Let $\varepsilon$ be a positive number, and let $C\left(L_{1}, L_{2}, \cdots, L_{n}\right)$ be a circular $\varepsilon / 3$-chain covering $M$. Then $M$ is covered by a tree $T$ such that

(1) each element of $T$ is a subset of a link of $C$,

(2) some element $K_{0}$ of $T$ intersects only one element of $C$, and 
(3) no element of $T$ intersects three elements of $C$. A function $f$ will be defined as follows over $T$. For each element $K$ of $T$, there is only one linear chain $\left(K_{0}, K_{1}, \cdots, K_{m}=K\right)$ from $K_{0}$ to $K$ in $T$. Let $f\left(K_{0}\right)=0$, and suppose that for some integer $i(0 \leq i \leq m), f\left(K_{i}\right)$ has been defined. Then define $f\left(K_{i+1}\right)$ as follows:

(1) let $f\left(K_{i+1}\right)=f\left(K_{i}\right)+1$ provided $K_{i}$ lies in some element $L_{\jmath}$ of $C$ and $K_{i+1}$ intersects $L_{j+1, \text { mod } n}$ but $K_{i}$ does not intersect this set,

(2) Let $f\left(K_{i+1}\right)=f\left(K_{i}\right)-1$ provided $K_{i+1}$ lies in some element $L_{j}$ of $C$ and $K_{i}$ intersects $L_{j+1, \bmod n}-L_{j}$ but $K_{i+1}$ does not intersect this set, and

(3) let $f\left(K_{i+1}\right)=f\left(K_{i}\right)$ provided neither (1) nor (2) is satisfied. The range of $f$ is an increasing finite sequence of consecutive integers $n_{1}, n_{2}$, $\cdots, n_{r}$. For each $t(1 \leq t \leq r)$, let $M_{t}$ denote the sum of all elements $X$ of $T$ such that $f(X)=n_{t}$. Then $\left(M_{1}, M_{2}, \cdots, M_{r}\right)$ is a linear $\varepsilon$-chain covering $M$.

\section{REFERENCES}

1. R. H. Bing, Concerning hereditarily indeconposable continua, Pacific, J. Math. 1 (1951), 43-51.

2. —- Snake-like continua, Duke Math. J. 18 (1951), 653-663.

3. - and F. B. Jones, Another homogencous plane continuum Trans. Amer. Math. Soc. 90 (1959), 171-192.

4. C. E. Burgess, Continua which are the sum of a finite number of indecomposable continua, Proc. Amer. Math. Soc. 4 (1953), 234-239.

5. - Separation properties and, n-indecomposable conlinua, Duke Math. J. 24 (1956), 595-600.

6. E. E. Moise, An indecomposable plane continuum which is homeomorphic to euch of its nondegenerate subcontinua, Trans. Amer. Math. Soc. 63 (1948), 581-594.

UNIVERSITY OF UTAH 



\section{PACIFIC JOURNAL OF MATHEMATICS}

\section{EDITORS}

\section{David Gilbarg}

Stanford University Stanford, California

\section{R. A. Beaumont}

University of Washington

Seattle 5, Washington

\section{A. L. Whiteman}

University of Southern California Los Angeles 7, California

L. J. Paige

University of California

Los Angeles 24, California

\section{ASSOCIATE EDITORS}

E. F. BECKENBACH
C. E. BURGESS
E. HEWITT
A. HORN

A. HORN

\author{
V. GANAPATHY IYER \\ R. D. JAMES \\ M. S. KNEBELMAN \\ L. NACHBIN
}

I. NIVEN

T. G. OSTROM

H. L. ROYDEN

M. M. SCHIFFER
E. G. STRAUS

G. SZEKERES

F. WOLF

K. YOSIDA

\section{SUPPORTING INSTITUTIONS}

\author{
UNIVERSITY OF BRITISH COLUMBIA \\ CALIFORNIA INSTITUTE OF TECHNOLOGY \\ UNIVERSITY OF CALIFORNIA \\ MONTANA STATE UNIVERSITY \\ UNIVERSITY OF NEVADA \\ OREGON STATE COLLEGE \\ UNIVERSITY OF OREGON \\ OSAKA UNIVERSITY \\ UNIVERSITY OF SOUTHERN CALIFORNIA
}

\author{
STANFORD UNIVERSITY \\ UNIVERSITY OF TOKYO \\ UNIVERSITY OF UTAH \\ WASHINGTON STATE COLLEGE \\ UNIVERSITY OF WASHINGTON \\ * * * * \\ AMERICAN MATHEMATICAL SOCIETY \\ CALIFORNIA RESEARCH CORPORATION \\ HUGHES AIRCRAFT COMPANY \\ SPACE TECHNOLOGY LABORATORIES
}

Mathematical papers intended for publication in the Pacific Journal of Mathematics should be typewritten (double spaced), and the author should keep a complete copy. Manuscripts may be sent to any one of the four editors. All other communications to the editors should be addressed to the managing editor, L. J. Paige at the University of California, Los Angeles 24, California.

50 reprints per author of each article are furnished free of charge; additional copies may be obtained at cost in multiples of 50 .

The Pacific Journal of Mathematics is published quarterly, in March, June, September, and December. The price per volume (4 numbers) is $\$ 12.00$; single issues, $\$ 3.50$. Back numbers are available. Special price to individual faculty members of supporting institutions and to individual members of the American Mathematical Society: $\$ 4.00$ per volume; single issues, $\$ 1.25$.

Subscriptions, orders for back numbers, and changes of address should be sent to Pacific Journal of Mathematics, 2120 Oxford Street, Berkeley 4, California.

Printed at Kokusai Bunken Insatsusha (International Academic Printing Co., Ltd.), No. 6, 2-chome, Fujimi-cho, Chiyoda-ku, Tokyo, Japan.

PUBLISHED BY PACIFIC IOURNAL OF MATHEMATICS, A NON-PROFIT CORPORATION

The Supporting Institutions listed above contribute to the cost of publication of this Journal, but they are not owners or publishers and have no responsibility for its content or policies. 


\section{Pacific Journal of Mathematics}

\section{Vol. 9, No. $3 \quad$ July, 1959}

Errett Albert Bishop, A minimal boundary for function algebras . . . . . . . . . . . . 629

John W. Brace, The topology of almost uniform convergence . . . . . . . . . . . . 643

Cecil Edmund Burgess, Chainable continua and indecomposability .......... 653

L. Carlitz, Multiplication formulas for products of Bernoulli and Euler

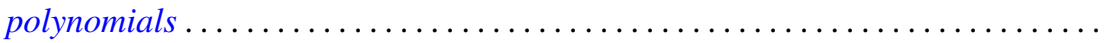

Eckford Cohen, A class of residue systems (mod $r$ ) and related arithmetical

functions. II. Higher dimensional analogues ....................

Shaul Foguel, Boolean algebras of projections of finite multiplicity . . . . . . . . . .

Richard Robinson Goldberg, Averages of Fourier coefficients .................

Seymour Goldberg, Ranges and inverses of perturbed linear operators .

Philip Hartman, On functions representable as a difference of convex functions ....

Milton Vernon Johns, Jr. and Ronald Pyke, On conditional expectation and

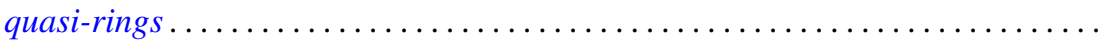

Robert Jacob Koch, Arcs in partially ordered spaces ....................

Gregers Louis Krabbe, A space of multipliers of type $L^{p}(-\infty, \infty) \ldots \ldots \ldots \ldots$

John W. Lamperti and Patrick Colonel Suppes, Chains of infinite order and their

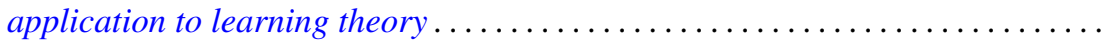

Edith Hirsch Luchins, On radicals and continuity of homomorphisms into Banach

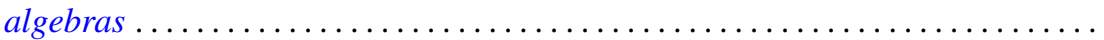

T. M. MacRobert, Multiplication formulae for the E-functions functions of their parameters.

Michael Bahir Maschler, Classes of minimal and representative domains and their kernel functions.

William Schumacher Massey, On the imbeddability of the real projective spaces in Euclidean space.

Thomas Wilson Mullikin, Semi-groups of class $\left(C_{0}\right)$ in $L_{p}$ determined by parabolic differential equations

Steven Orey, Recurrent Markov chains

Ernest Tilden Parker, On quadruply transitive groups ........ . .

Calvin R. Putnam, On Toeplitz matrices, absolute continuity, and unitary

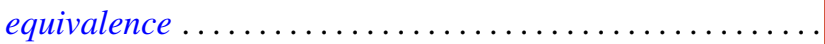

Helmut Heinrich Schaefer, On nonlinear positive operators.

Robert Seall and Marion Wetzel, Some connections between continued fractions and convex sets

Robert Steinberg, Variations on a theme of Chevalley

Olga Taussky and Hans Zassenhaus, On the similarity transformation between a

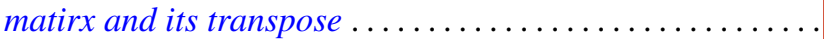

Emery Thomas, The suspension of the generalized Pontrjagin cohomology

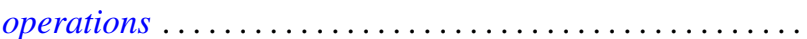

Joseph L. Ullman, On Tchebycheff polynomials ..................... 913

Richard Steven Varga, Orderings of the successive overrelaxation scheme ........ 925

Orlando Eugenio Villamayor, Sr., On weak dimension of algebras . 